CLINICAL STUDY

\title{
Validity and reliability of the novel thyroid-specific quality of life questionnaire, ThyPRO
}

\author{
Torquil Watt ${ }^{1,2}$, Laszlo Hegedüs ${ }^{3}$, Mogens Groenvold ${ }^{2,4}$, Jakob Bue Bjorner ${ }^{2,5}$, Åse Krogh Rasmussen ${ }^{1}$, \\ Steen Joop Bonnema ${ }^{3}$ and Ulla Feldt-Rasmussen ${ }^{1}$ \\ ${ }^{1}$ Department of Endocrinology, Copenhagen University Hospital Rigshospitalet, Blegdamsvej 9, DK-2100 Copenhagen Ø, Denmark, ${ }^{2}$ Institute of Public \\ Health, University of Copenhagen, DK-1014 Copenhagen, Denmark, ${ }^{3}$ Department of Endocrinology and Metabolism, Odense University Hospital, Odense, \\ Denmark, ${ }^{4}$ Department of Palliative Medicine, Bispebjerg Hospital, Copenhagen, Denmark and ${ }^{5}$ National Research Centre for the Working Environment, \\ Copenhagen, Denmark
}

(Correspondence should be addressed to T Watt at Department of Endocrinology, Copenhagen University Hospital Rigshospitalet; Email: t.watt@rh.dk)

\begin{abstract}
Background: Appropriate scale validity and internal consistency reliability have recently been documented for the new thyroid-specific quality of life (QoL) patient-reported outcome (PRO) measure for benign thyroid disorders, the ThyPRO. However, before clinical use, clinical validity and test-retest reliability should be evaluated.

Aim: To investigate clinical ('known-groups') validity and test-retest reliability of the Danish version of the ThyPRO.

Methods: For each of the 13 ThyPRO scales, we defined groups expected to have high versus low scores ('known-groups'). The clinical validity (known-groups validity) was evaluated by whether the ThyPRO scales could detect expected differences in a cross-sectional study of 907 thyroid patients. Test-retest reliability was evaluated by intra-class correlations of two responses to the ThyPRO 2 weeks apart in a subsample of 87 stable patients.

Results: On all 13 ThyPRO scales, we found substantial and significant differences between the groups expected to have high versus low scores. Test-retest reliability was above 0.70 (range $0.77-0.89$ ) for all scales, which is usually considered necessary for comparisons among patient groups, but below 0.90 , which is the usual threshold for use in individual patients.

Conclusion: We found support for the clinical validity of the new thyroid-specific QoL questionnaire, ThyPRO, and evidence of good test-retest reliability. The questionnaire is now ready for use in clinical studies of patients with thyroid diseases.
\end{abstract}

European Journal of Endocrinology 162 161-167

\section{Introduction}

Measurements applying standardized self-reports to capture the impact of health on patients' lives are termed health-related quality of life (HRQL) measurements (1). They usually conceptualize HRQL as a multidimensional concept encompassing various aspects of physical, mental, and social functioning and well-being. To an increasing extent, the broader, but also more neutral term 'patient-reported outcomes (PROs)' is replacing HRQL. Today, PROs or HRQL measurements are recognized as inevitable and important outcomes in high quality clinical studies. Further, they can provide important documentation for evidence-based patient information and may even be implemented in clinical management of the individual patient, as has been done within, e.g. oncology (2), where randomized trials have documented significant improvement of patient-clinician interaction, without prolonging consultations, and impact on patient management (3). HRQL measurements may be either generic, i.e. applicable to any patient group regardless of diagnosis, or specific, i.e. targeted to a specific disease group. Specific HRQL measurements are usually more sensitive than generic, which on the other hand have the advantage of allowing comparisons across dissimilar populations.

Some questionnaires have been developed for specific thyroid diseases (4-12). However, a thoroughly validated questionnaire only exists for thyroid-associated ophthalmopathy (TAO) patients (5-7). Another TAO questionnaire has been developed, but has not been validated (8). One questionnaire for patients with hyperthyroidism was developed, but has never been validated (4). Three questionnaires for hypothyroid patients have been developed (9-12), but studies 
evaluating the validity of these measures are still awaited. Most importantly, no validated, thyroid-specific PRO instrument is available for use across different thyroid diseases (13). This is a major deficiency, because benign thyroid diseases are characterized by a substantial overlap between various disease entities (e.g. coexistence of goitre and hyperthyroidism) and a shift between diseases (e.g. hyperthyroid patients becoming hypothyroid through ablative therapy). Therefore, optimally an HRQL outcome measure for thyroid patients should encompass all thyroid diseases in order to have content validity (i.e. capture HRQL issues of relevance to the patients). If not, the results of longitudinal studies may be misleading, because important HRQL aspects are not measured at follow-up (e.g. impact of hypothyroidism after ablative therapy).

We have recently developed a quality of life (QoL) questionnaire for patients with benign thyroid diseases, called the ThyPRO (13-15), and evaluated important aspects of its measurement properties (16). In HRQL terminology, the measurement property termed accuracy within biochemical assay methodology (i.e. degree of systematic bias) is called validity and what endocrinologists may refer to as precision (or reproducibility) is termed reliability. In the just mentioned validation study (16), support for appropriate validity and reliability was found, in terms of a valid scale structure (an important aspect of what is termed construct validity) and very good internal consistency reliability. However, for HRQL measurements, evaluation of accuracy, or validity, is a more complex task than with most other measurement fields, since no gold standard against which other measures can be tested is available. Thus, the validation of an HRQL measure is an iterative process where evidence for or against the validity of a measure is usually gathered by several studies taking different approaches to evaluating validity, one of which is the above mentioned scale validation. Another important way of assessing validity, especially for measures attempted for clinical use, is termed known-groups validity. In this approach, clinically based criteria are used to classify patients into groups with expected high or low scores on a questionnaire and then test whether these expected differences are found in patient samples. Precision, or reliability, can also be evaluated by several techniques. One approach is 'internal consistency reliability' (Cronbach's alpha), which has been used in the initial analyses of the ThyPRO (16). Another approach is testretest reproducibility where duplicate measurements are obtained by collecting two responses from stable respondents separated by 2-3 weeks (17).

The purpose of the present study was to investigate accuracy, in terms of clinical/known-groups validity, and precision, in terms of test-retest reliability, of the Danish version of the thyroid-specific QoL questionnaire, the ThyPRO.

\section{Material and methods}

\section{Patients and clinical characterization}

Patients were recruited from the endocrinological outpatient clinics at two university hospitals in Denmark: Copenhagen University Hospital Rigshospitalet $(\mathrm{RH})$ and Odense University Hospital (OUH). At $\mathrm{RH}$, the sampling strategy was cross-sectional: all thyroid patients born within the first 20 days of each month (to limit running sample size) were invited during February-June 2007 by mail 3 weeks prior to their appointment in the clinic. At $\mathrm{OUH}$, all eligible patients referred to the thyroid unit of the endocrine outpatient clinic during May-November 2007 were recruited. The questionnaire was sent about 3 weeks prior to the appointment in the clinic. Blood samples were drawn the week prior to their appointment, and the participants were instructed to complete the questionnaire at about that time. Questionnaires were either returned by mail or delivered by hand in the laboratory or at the clinic on the day of appointment. Exclusion criteria were absence of any thyroid disorder, thyroid cancer, age $<15$, and inability to complete a questionnaire due to communication problems (non-Danish speaking, blindness, etc). One reminder was sent after 2 weeks to non-responders, and all participants gave signed informed consent.

Socio-demographic data and information about co-morbidity and non-thyroid medication were selfreported. Laboratory data, diagnostic imaging results, exact diagnosis, previous and current treatment and time of diagnosis among respondents were obtained by chart review. Biochemical thyroid tests were TSH, total thyroxine $\left(\mathrm{T}_{4}\right)$, total triiodothyronine $\left(\mathrm{T}_{3}\right)$, free $\mathrm{T}_{4}\left(\mathrm{FT}_{4}\right)$, free $\mathrm{T}_{3}\left(\mathrm{FT}_{3}\right)$, resin- $\mathrm{T}_{3}$ test (only $\mathrm{OUH}$ ), thyroid peroxidase antibodies (TPOAb), thyroglobulin antibodies (only in patients diagnosed with hypothyroidism and negative TPOAb) and TSH receptor antibodies (only in patients with a diagnosis of hyperthyroidism). All analyses were performed using the standard methods at the laboratories of the participating hospitals. In patients with thyroid eye disease TAO, NOSPECS (18) classification was performed by an ophthalmologist and clinical activity scoring (19) was performed by a physician. Thyroid volume was determined by ultrasound, using the ellipsoid method (20). All examiners were blinded to the ThyPRO results. The patients were classified according to primary diagnosis, i.e. their initial diagnosis, prior to treatment. For example, patients with a non-toxic goitre, who had their thyroid removed and thus have hypothyroidism and receive thyroid hormone replacement, were classified with a diagnosis of non-toxic goitre. Self-completed data were entered using optical scanning. All clinical data were entered via SPSS Data Entry Builder 4.0 (SPSS Inc, Chicago, IL, USA) by medical staff. Data were converted into 
SAS datasets, and all analyses were performed with SAS 9.1 (SAS Institute, Cary, NC, USA). The project was approved by the local ethical committee (KF01 2006-1579) and the Danish data protection agency and registered at ClinicalTrials.gov (NCT00150033).

\section{Outcome}

The ThyPRO questionnaire is self-administered and measures QoL with 13 scales (see Table 1), covering physical and mental symptoms, well-being and function as well as impact of thyroid disease on participation (i.e. social and daily life) and overall QoL. It consists of 84 items and, on average, takes $14 \mathrm{~min}$ to complete. Each scale ranges $0-100$ with increasing scores indicating decreasing QoL (i.e. more symptoms or greater impact of disease) (16).

\section{Known groups}

Groups with expected high and low scores were defined a priori by a panel of four thyroid experts. The criteria used for this classification is outlined in Table 1. They include clinical data, physician ratings of overall clinical condition, and depression and anxiety status according to Hospital Anxiety and Depression Scale (HADS). The overall physician ratings were obtained at the outpatient visit, where the consulting physician, who was blinded to the ThyPRO results, rated the overall clinical condition of the patient on a five-point scale ranging from 'very bad' to 'excellent', based on all available clinical information, including patient history. Depression or anxiety according to HADS was scored using its standard thresholds: a score $<8$ on the depression scale indicates absence of depression, and a score above 10 indicates depression, and likewise for the anxiety scale. In addition, we evaluated the correlation between the ThyPRO depressivity and anxiety scales and the HADS depression and anxiety scales respectively using both parametric (Pearson) and non-parametric (Spearman) correlation.

\section{Test-retest data}

Since fewer patients were required for test-retest analyses than for the other validation analyses, only patients enrolled at RH during a limited period (midApril to mid-June 2007) were included in the test-retest study. The only difference compared to the main study was the fact that they were asked to complete another questionnaire about 2 weeks after their first response. In addition, they rated any change occurring since the initial response on a seven-point scale ("compared to when you answered the first time, would you say your overall state is much worse/somewhat worse/a little worse/more or less the same/a little better/somewhat

Table 1 Description of the expected high versus low score groups used in the known groups comparisons.

\begin{tabular}{|c|c|c|}
\hline Scale & Expected high score group & Expected low score group \\
\hline Goitre symptoms & $\begin{array}{l}\text { Patients with untreated non-toxic diffuse or } \\
\text { multinodular goitre }(n=105)\end{array}$ & $\begin{array}{l}\text { Patients with non-goitrous autoimmune } \\
\text { hypothyroidism treated with L-thyroxine } \\
\text { for at least } 3 \text { months }(n=107)\end{array}$ \\
\hline Hyperthyroid symptoms & $\begin{array}{l}\text { Patients with hyperthyroid Graves' disease or } \\
\text { nodular goitre and overt hyperthyroidism }(n=70)\end{array}$ & $\begin{array}{l}\text { Untreated non-toxic diffuse or nodular } \\
\text { goitre }(n=161)\end{array}$ \\
\hline Hypothyroid symptoms & $\begin{array}{l}\text { Patients diagnosed with overt hypothyroidism } \\
\text { within the last } 3 \text { months }(n=20)\end{array}$ & $\begin{array}{l}\text { Untreated non-toxic diffuse or } \\
\text { nodular goitre }(n=161)\end{array}$ \\
\hline Eye symptoms & $\begin{array}{l}\text { Patients with TAO and NOSPECS }>1 \\
\text { (i.e. worse than 'Only signs') }(n=16)\end{array}$ & $\begin{array}{l}\text { Untreated non-toxic diffuse or nodular } \\
\text { goitre }(n=161)\end{array}$ \\
\hline Tiredness & $\begin{array}{l}\text { Patients with overall clinical condition } \\
\text { rated by physician as 'very bad' }(n=12)\end{array}$ & $\begin{array}{l}\text { Patients with overall clinical condition } \\
\text { rated by physician as 'excellent' }(n=88)\end{array}$ \\
\hline Cognitive impairment & $\begin{array}{l}\text { Patients with overall clinical condition } \\
\text { rated by physician as 'very bad' }(n=12)\end{array}$ & $\begin{array}{l}\text { Patients with overall clinical condition rated by } \\
\text { physician as 'excellent' }(n=87)\end{array}$ \\
\hline Anxiety & $\begin{array}{l}\text { Patients with a HADS anxiety score indicating } \\
\text { anxiety (i.e. score }>10)(n=146)\end{array}$ & $\begin{array}{l}\text { Patients with a HADS anxiety score indicating } \\
\text { no anxiety (i.e. score }<8)(n=577)\end{array}$ \\
\hline Emotional susceptibility & $\begin{array}{l}\text { Patients with overall clinical condition rated by } \\
\text { physician as 'very bad' }(n=12)\end{array}$ & $\begin{array}{l}\text { Patients with overall clinical condition rated by } \\
\text { physician as 'excellent' }(n=87)\end{array}$ \\
\hline Impaired social life & $\begin{array}{l}\text { Patients with overall clinical condition rated by } \\
\text { physician as 'very bad' }(n=12)\end{array}$ & $\begin{array}{l}\text { Patients with overall clinical condition rated by } \\
\text { physician as 'excellent' }(n=84)\end{array}$ \\
\hline Impaired daily life & $\begin{array}{l}\text { Patients with overall clinical condition rated by } \\
\text { physician as 'very bad' }(n=12)\end{array}$ & $\begin{array}{l}\text { Patients with overall clinical condition rated by } \\
\text { physicians as 'excellent' }(n=85)\end{array}$ \\
\hline Impaired sex life & $\begin{array}{l}\text { Patients with overall clinical condition rated by } \\
\text { physician as 'very bad' }(n=9)\end{array}$ & $\begin{array}{l}\text { Patients with overall clinical condition rated by } \\
\text { physician as 'excellent' }(n=85)\end{array}$ \\
\hline Cosmetic complaints & $\begin{array}{l}\text { Patients with TAO and NOSPECS above } 1 \text { and } \\
\text { patients with non-toxic diffuse or multinodular } \\
\text { goitre with a volume greater than } 150 \mathrm{ml}(n=21)\end{array}$ & $\begin{array}{l}\text { Patients with autoimmune hypothyroidism and } \\
\text { an overall clinical condition rated by physician } \\
\text { as 'excellent' }(n=22)\end{array}$ \\
\hline
\end{tabular}

TAO, thyroid-associated ophthalmopathy; NOSPECS, a classification system for defining severity of TAO; HADS, Hospital Anxiety and Depression Scale. 
better/much better'). Patients who completed the second questionnaire between 10 and 24 days after the first, and who rated themselves as stable were included in test-retest analyses.

\section{Statistical analyses}

Differences in mean scale scores between the expected high versus low level groups were analysed with Student's unpaired $t$-test, with Satterthwaites correction in case of unequal variances according to the folded $F$ test, using SAS PROC TTEST.

Test-retest reliability was evaluated by intra-class correlations between the two measurements (21-24). Correlations were calculated using SAS PROC GLM, and 95\% confidence intervals were estimated by empirical bootstrap (25, SV Thorsen and JB Bjorner, unpublished observations).

\section{Results}

In total, 907 responses from 1316 eligible patients were obtained, yielding an overall response rate of $69 \%$, as detailed elsewhere (16). Of these, 195 were included in the test-retest study, $149(76 \%)$ of whom returned a second response. Eighty-seven of the 195 fulfilled the criteria for inclusion in the analyses (Fig. 1).

Clinical characteristics for the total sample as well as for the test-retest subsample are given in Table 2 .

As seen in Fig. 2, the expected high level groups had substantially higher mean scores compared with the expected low level groups on all ThyPRO scales.

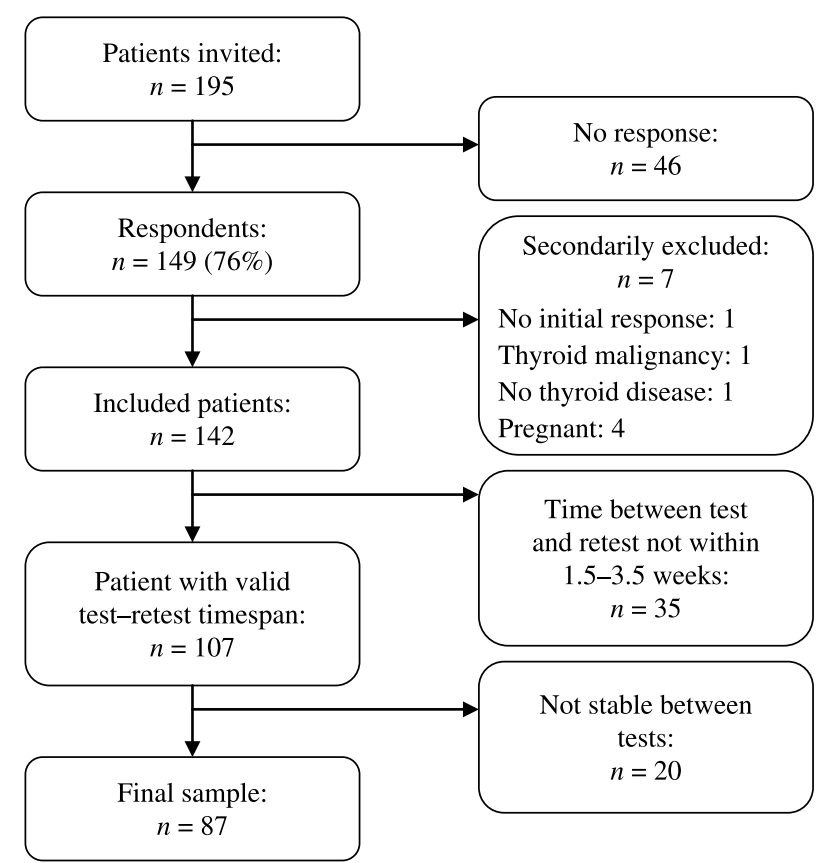

Figure 1 Derivation of the test-retest subsample.
Table 2 Patient characterization. Clinical characteristics are presented for the total sample and for the test-retest subsample.

\begin{tabular}{|c|c|c|}
\hline & Total sample & $\begin{array}{l}\text { Test-retest } \\
\text { subsample }\end{array}$ \\
\hline Sample size & 907 & 87 \\
\hline Women (\%)/men & $787(87) / 120$ & $81(93) / 6$ \\
\hline Age (mean (S.D.)) & $51(15)$ & 49 (13) \\
\hline \multicolumn{3}{|l|}{ Diagnosis $(n(\%))$} \\
\hline Diffuse non-toxic goitre & $18(2)$ & $1(1)$ \\
\hline Multinodular non-toxic goitre & $154(17)$ & $12(14)$ \\
\hline Uninodular non-toxic goitre & $68(7)$ & $2(2)$ \\
\hline Solitary cyst & $19(2)$ & \\
\hline Multinodular toxic goitre & $108(12)$ & $3(3)$ \\
\hline Uninodular toxic goitre & $37(4)$ & $5(6)$ \\
\hline Graves' disease & $168(19)$ & $28(32)$ \\
\hline TAO & $94(10)$ & $9(10)$ \\
\hline Autoimmune hypothyroidism & $199(22)$ & $24(28)$ \\
\hline Subacute thyroiditis & $9(1)$ & \\
\hline Post-partum thyroiditis & $8(1)$ & $1(1)$ \\
\hline Other thyroid disease & $25(3)$ & $2(2)$ \\
\hline $\begin{array}{l}\text { Months since diagnosis } \\
(\text { median (range) }))^{a}\end{array}$ & $27(-0.9-607)$ & $47(0.7-269)$ \\
\hline \multicolumn{3}{|l|}{ Thyroid treatment $(n(\%))$} \\
\hline No thyroid treatment (ever) & $283(31)$ & $10(11)$ \\
\hline Antithyroid medication & $162(18)$ & $20(23)$ \\
\hline L-thyroxine & $292(32)$ & $43(50)$ \\
\hline Radioiodine & $114(13)$ & $14(16)$ \\
\hline Thyroidectomy & $132(14)$ & $8(10)$ \\
\hline Other treatment & $4(0.4)$ & 0 \\
\hline \multicolumn{3}{|c|}{ Current thyroid function ${ }^{\mathrm{b}}(n(\%))$} \\
\hline Euthyroid & $530(58)$ & $60(70)$ \\
\hline Mildly hypothyroid & $124(14)$ & $8(9)$ \\
\hline Overtly hypothyroid & $15(2)$ & 0 \\
\hline Mildly hyperthyroid & $122(13)$ & $16(19)$ \\
\hline Overtly hyperthyroid & $98(11)$ & $2(2)$ \\
\hline Positive TPOAb (n (\%)) & $367(46)$ & $55(64)$ \\
\hline Positive TSHRAb $(n(\%))$ & $174(35)$ & $22(37)$ \\
\hline $\begin{array}{l}\text { Thyroid volume } \\
\text { (mean (s.D.), ml) }\end{array}$ & $26(32)$ & $25(25)$ \\
\hline
\end{tabular}

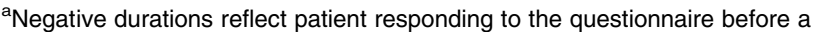
final thyroid diagnosis was established.

${ }^{b}$ Euthyroid, normal TSH; mildly hypothyroid, elevated TSH and normal $\mathrm{T}_{4}$; overtly hypothyroid, elevated TSH and decreased $\mathrm{T}_{4}$; mildly hyperthyroid, decreased TSH and normal $\mathrm{T}_{4}$ and $\mathrm{T}_{3}$; overtly hyperthyroid, decreased TSH and elevated $T_{4}$ or $T_{3}$.

All differences were statistically significant using unpaired $t$-tests $(P<0.001$ for all scales except hypothyroid symptoms and cosmetic complaints, where $P<0.05)$. The correlations between ThyPRO and HADS scales were 0.72 for depressivity/depression and 0.77 for anxiety $(P<0.0001$ for both), both with parametric and non-parametric methods.

In the test-retest analyses, all intra-class correlations were above 0.70 (Table 3 ) and all but two (anxiety (0.77) and hypothyroid symptoms (0.80)) scales were above the 'almost perfect' concordance (i.e. >0.81) (26).

\section{Discussion}

The purpose of the present study was to investigate clinical known-groups validity and test-retest reliability of the Danish version of the thyroid-specific QoL questionnaire, the ThyPRO. 


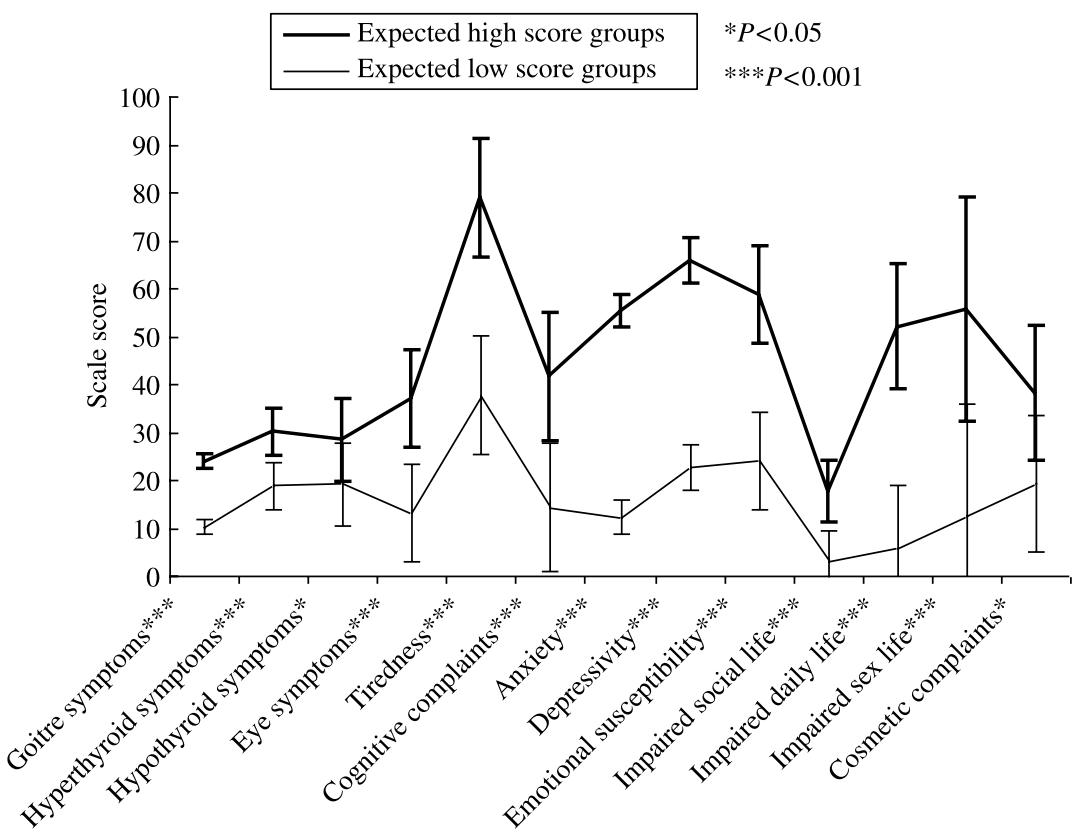

Figure 2 Mean scale scores for the expected high score groups versus the low score groups for the 13 ThyPRO scales. Vertical lines indicate $95 \%$ confidence intervals of the means.
When comparing specified subgroups of patients expected to have high scale scores on each of the 13 QoL scales to subgroups expected to have low scores, we found significant differences on all scales, supporting the clinical validity of the ThyPRO. This is an important and encouraging finding.

The magnitudes of these differences varied: differences on the symptom scales, the cosmetic complaints scale and the impaired social life scale were smaller than the rest. The simple conclusion that these scales are just less sensitive (i.e. are less efficient in detecting differences among groups) can be elaborated by a closer look at the criteria used to define these groups. In fact, the analyses of these scales differed from the rest, in that the criteria defining the high versus low score groups were entirely clinical and biochemical, except for the impaired daily life scale. Criteria for the other scales were based on the consulting clinician's rating of the overall clinical condition or HADS anxiety and depression scores. To better understand these differences, it is useful to consider a theoretical model for health outcomes. Based on the WHO ICF framework (27) and on the conceptual model for health outcomes proposed by Wilson \& Cleary (28), we propose to distinguish four levels of health outcomes: i) biological and physiological variables, ii) symptoms and signs, iii) functioning, i.e. what you can physically do, and iv) participation, i.e. how you function in your social environment. Regarding the symptom scales, the criteria used to define the groups for comparisons relate to biological and physiological variables, whereas the scale scores reflect patients' experiences of symptoms and signs. The same is true for the cosmetic complaints scale. Thus, since the disease group comparisons do not directly concern the same level of health outcomes as the ThyPRO scales, it is reasonable that the associations are fairly small. On the other hand, the overall clinical rating used for evaluating the other scales, except anxiety and depressivity, is likely to be based on symptoms and signs, and functioning, as well as diagnosis and physiological measures. Thus, strong associations for these scales are likely, which is also what we found, except for the impaired social life scale, which reflects participation rather than functioning. Finally, we are likely to see strong associations between ThyPRO scales for anxiety and depression, and other scales assessing the same domains, i.e. HADS.

For all scales, test-retest reliability was above standard thresholds for adequate reliability, indicating that the measures have appropriate reliability for use in clinical studies (29). Since the test-retest reliabilities found in this study were a little lower than the

Table 3 Intra-class correlation coefficients with 95\% confidence intervals $(\mathrm{Cl})$ between the two measurements in the test-retest subsample.

\begin{tabular}{lc}
\hline Scale & $\begin{array}{c}\text { Intra-class correlation } \\
\text { coefficient }(95 \% \mathrm{Cl})\end{array}$ \\
\hline Goitre symptoms & $0.87(0.81-0.91)$ \\
Hyperthyroid symptoms & $0.89(0.82-0.93)$ \\
Hypothyroid symptoms & $0.80(0.71-0.87)$ \\
Eye symptoms & $0.86(0.77-0.92)$ \\
Tiredness & $0.85(0.77-0.91)$ \\
Cognitive complaints & $0.88(0.79-0.93)$ \\
Anxiety & $0.77(0.64-0.88)$ \\
Depressivity & $0.88(0.82-0.93)$ \\
Emotional susceptibility & $0.87(0.80-0.91)$ \\
Impaired social life & $0.84(0.72-0.91)$ \\
Impaired daily life & $0.83(0.71-0.91)$ \\
Impaired sex life & $0.86(0.74-0.94)$ \\
Cosmetic complaints & $0.85(0.79-0.91)$ \\
\hline
\end{tabular}


coefficients found by internal consistency reliability (16), the test-retest reliabilities may even underestimate true reliability slightly, possibly due to less than perfect clinically stable conditions of the patients in the test interval. In fact, the mean score was indeed a little lower at time of re-evaluation, for all scales. No scale achieved a reliability coefficient above the traditional cut-point of 0.90 required for scales used with individual patients.

Since this is the first QoL measure for use in thyroid patients covering the majority of benign thyroid disorders, no previous studies evaluating measurement properties of such measures exist. Regarding individual diagnoses, test-retest reliability and clinical known groups validity have been evaluated (6) for a diseasespecific questionnaire for patients with TAO. Test-retest reliability estimates were comparable to the ones found in the present study. However, their clinical validity analyses did not entirely support the validity of the questionnaire, since some of the expected associations with clinical variables were not found (6). The interpretations of these lacking associations offered by the authors are analogous to our interpretations regarding the criteria for evaluations of the symptom scales. They suggested that it was due to the fact that the clinical variables defining the groups related to health outcomes (i.e. biological and physiological variables) differed from the health outcomes relating to QoL (i.e. symptoms, functioning and participation). For another TAO questionnaire, no associations with clinical variables were found at all (8). To our knowledge, no other study has evaluated clinical validity or test-retest reliability of any of the other existing thyroid-specific (i.e. for individual diagnoses) questionnaires (reviewed in (13)).

Among the strengths of our study is a large sample size, enabling a detailed analysis of each group of benign thyroid disorders with adequate power. Moreover, the patients are clinically well characterized, especially compared to other studies of generic or specific QoL in thyroid patients (13). The fact that each patient was rated by a physician at about the same time that they completed the questionnaire is an important strength of the clinical validity analyses. This is also true for the availability of a 'stability-measure' for identification of patients to enter the test-retest substudy. Another strength is the fact that we used only few exclusion criteria, ensuring validity of the questionnaire also for subpopulations with e.g. psychiatric co-morbidities, etc. Of course, future descriptive studies evaluating HRQL in thyroid patients may consider excluding these patient groups in order to limit the description to only impairments related to thyroid diseases.

One limitation of the study relates to its design: due to our cross-sectional design, our analyses of clinical validity are limited to cover differences between groups. Ongoing studies are evaluating the ability of the questionnaire to detect relevant changes in patients over time (responsiveness) and the minimal important differences in scores. However, compared to other disease-specific instruments, including those covering other disease areas than thyroid diseases, the ThyPRO is already very well validated. Another limitation relates to the clinician evaluation. Although it is of value that these are available for each individual patient at time of survey completion, the fact that they are also based on patient history implies that they are not entirely external criteria, as are for example thyroid function tests, etc. Still, they do represent evaluations external to the ThyPRO, and clinician evaluations are often used in studies of criterion validity $(30,31)$.

Although the patients are clinically well characterized, and a large number of clinical descriptors are available at time of completion, the timing is not perfect; some patients may have had blood samples drawn up to about 3 weeks away from their questionnaire response. However, this would weaken the associations between e.g. thyroid function tests and HRQL data, but we still find positive associations despite such hypothetical bias.

Information from clinical known-groups studies, responsiveness studies and item response theory analyses could be utilized in an attempt to develop shorter versions of the ThyPRO by identifying the best performing items to be included in such abbreviated outcome measures. Ongoing studies are ensuring crosscultural validity of this measure in seven languages, including English, and versions in further languages are being planned.

We recommend the use of the ThyPRO measure in studies evaluating important clinical questions regarding therapy of thyroid patients, such as whether patients with mild thyroid disease benefit from treatment (32) and whether block replacement therapy with antithyroid drugs and levothyroxine is associated with a better QoL in patients with hyperthyroidism than monotherapy with antithyroid drugs (33).

In conclusion, all scales of the ThyPRO detected clinically relevant differences among thyroid patients, and the ThyPRO was found to have adequate test-retest reliability.

\section{Declaration of interest}

The authors declare that there is no conflict of interest that could be perceived as prejudicing the impartiality of the research reported.

\section{Funding}

This study has been supported by grants from the Danish Medical Research Council, Agnes and Knut Mørk's Foundation, Aase and Ejnar Danielsen's Foundation, Else and Mogens Wedell-Wedellsborg's Foundation, the Genzyme Corporation, the Novo Nordisk Foundation and the Danish Thyroid Foundation.

\section{Acknowledgements}

We wish to express our gratitude to the staffs and colleagues at Department of Endocrinology at Rigshospitalet as well as at Odense University Hospital. 


\section{References}

1 Guyatt GH, Feeny DH \& Patrick DL. Measuring health-related quality of life. Annals of Internal Medicine 1993118 622-629.

2 Sloan JA, Frost MH, Berzon R, Dueck A, Guyatt G, Moinpour C, Sprangers M, Ferrans C \& Cella D. The clinical significance of quality of life assessments in oncology: a summary for clinicians. Supportive Care in Cancer 200614 988-998.

3 Velikova G, Booth L, Smith AB, Brown PM, Lynch P, Brown JM \& Selby PJ. Measuring quality of life in routine oncology practice improves communication and patient well-being: a randomized controlled trial. Journal of Clinical Oncology 200422 714-724.

4 Fahrenfort JJ, Wilterdink AM \& van der Veen EA. Long-term residual complaints and psychosocial sequelae after remission of hyperthyroidism. Psychoneuroendocrinology 200025 201-211.

5 Terwee CB, Gerding MN, Dekker FW, Prummel MF \& Wiersinga WM. Development of a disease specific quality of life questionnaire for patients with Graves' ophthalmopathy: the GO-QOL. British Journal of Ophthalmology $1998 \mathbf{8 2} 773-779$.

6 Terwee CB, Gerding MN, Dekker FW, Prummel MF, van der Pol JP \& Wiersinga WM. Test-retest reliability of the GO-QOL: a disease-specific quality of life questionnaire for patients with Graves' ophthalmopathy. Journal of Clinical Epidemiology 1999 52 875-884.

7 Terwee CB, Dekker FW, Mourits MP, Gerding MN, Baldeschi L, Kalmann R, Prummel MF \& Wiersinga WM. Interpretation and validity of changes in scores on the Graves' ophthalmopathy quality of life questionnaire (GO-QOL) after different treatments. Clinical Endocrinology 200154 391-398.

8 Tehrani M, Krummenauer F, Mann WJ, Pitz S, Dick HB \& Kahaly GJ. Disease-specific assessment of quality of life after decompression surgery for Graves' ophthalmopathy. European Journal of Ophthalmology 200414 193-199.

9 Jaeschke R, Guyatt G, Gerstein H, Patterson C, Molloy W, Cook D, Harper S, Griffith L \& Carbotte R. Does treatment with L-thyroxine influence health status in middle-aged and older adults with subclinical hypothyroidism? Journal of General Internal Medicine $199611744-749$.

10 Jaeschke R, Guyatt G, Cook D, Harper S \& Gerstein HC. Spectrum of quality of life impairment in hypothyroidism. Quality of Life Research 19943 323-327.

11 Saravanan P, Chau WF, Roberts N, Vedhara K, Greenwood R \& Dayan CM. Psychological well-being in patients on 'adequate' doses of L-thyroxine: results of a large, controlled community-based questionnaire study. Clinical Endocrinology 200257 577-585.

12 McMillan CV, Bradley C, Woodcock A, Razvi S \& Weaver JU. Design of new questionnaires to measure quality of life and treatment satisfaction in hypothyroidism. Thyroid 2004 14 916-925.

13 Watt T, Groenvold M, Rasmussen AK, Bonnema SJ, Hegedüs L, Bjorner JB \& Feldt-Rasmussen U. Quality of life in patients with benign thyroid disorders. A review. European Journal of Endocrinology 2006154 501-510.

14 Watt T, Hegedüs L, Rasmussen AK, Groenvold M, Bonnema SJ. Bjorner JB \& Feldt-Rasmussen U. Which domains of thyroidrelated quality of life are most relevant? Patients and clinicians provide complementary perspectives Thyroid $2007 \mathbf{1 7} 647-654$.

15 Watt T, Rasmussen AK, Groenvold M, Bjorner JB, Watt SH, Bonnema SJ, Hegedüs L \& Feldt-Rasmussen U. Improving a newly developed patient-reported outcome for thyroid patients, using cognitive interviewing. Quality of Life Research $2008 \mathbf{1 7}$ 1009-1017.
16 Watt T, Bjorner JB, Groenvold M, Rasmussen AK, Bonnema SJ, Hegedüs L \& Feldt-Rasmussen U. Establishing construct validity for the thyroid-specific patient reported outcome measure (ThyPRO): an initial examination. Quality of Life Research 2009 $18483-496$.

17 Streiner DL \& Norman GR. Health measurement scales. In $A$ Practical Guide to their Development and Use, edn 4. Oxford: Oxford University Press, 2008.

18 Werner SC. Modification of the classification of the eye changes of Graves' disease: recommendations of the Ad Hoc Committee of the American Thyroid Association. Journal of Clinical Endocrinology and Metabolism 197744 203-204.

19 Mourits MP, Prummel MF, Wiersinga WM \& Koornneef L. Clinical activity score as a guide in the management of patients with Graves' ophthalmopathy. Clinical Endocrinology 1997 47 9-14.

20 Hegedüs L. Thyroid ultrasound. Endocrinology and Metabolism Clinics of North America 200130 339-360.

21 Tammemagi MC, Frank JW, Leblanc M, Artsob H \& Streiner DL. Methodological issues in assessing reproducibility - a comparative study of various indices of reproducibility applied to repeat ELISA serologic tests for Lyme disease. Journal of Clinical Epidemiology 199548 1123-1132.

22 Shrout PE \& Fleiss JL. Intraclass correlations: uses in assessing rater reliability. Psychological Bulletin 197986 420-428.

23 Deyo RA, Diehr P \& Patrick DL. Reproducibility and responsiveness of health status measures. Statistics and strategies for evaluation. Controlled Clinical Trials 199112 142S-158S.

24 Fayers PM \& Machin D. Quality of Life: Assessment, Analysis and Interpretation Chichester: John Wiley and Sons, 2000.

25 Henderson AR. The bootstrap: a technique for data-driven statistics. Using computer-intensive analyses to explore experimental data. Clinica Chimica Acta 2005359 1-26.

26 Landis JR \& Koch GG. The measurement of observer agreement for categorical data. Biometrics 197733 159-174.

27 World Health Organization. International Classification of Functioning, Disability, and Health Geneva: World Health Organization, 2001.

28 Wilson IB \& Cleary PD. Linking clinical variables with healthrelated quality of life. A conceptual model of patient outcomes. Journal of the American Medical Association 1995273 59-65.

29 Nunnally JC \& Bernstein I. Psychometric Theory, edn 3. New York: McGraw-Hill, 1994.

30 Schatz M, Sorkness CA, Li JT, Marcus P, Murray JJ, Nathan RA, Kosinski M, Pendergraft TB \& Jhingran P. Asthma control test: reliability, validity, and responsiveness in patients not previously followed by asthma specialists. Journal of Allergy and Clinical Immunology 2006117 549-556.

31 Miller IW, Epstion NB, Bishop DS \& Keitner GI. The McMaster family assessment device: reliability and validity. Journal of Marital and Family Therapy 200911 345-356.

32 Biondi B \& Cooper DS. The clinical significance of subclinical thyroid dysfunction. Endocrine Reviews 200829 76-131.

33 Hegedüs L. Treatment of Graves' hyperthyroidism: evidence-based and emerging modalities. Endocrinology and Metabolism Clinics of North America 200938 355-371.

Received 25 September 2009

Accepted 1 October 2009 